University of Nebraska - Lincoln

DigitalCommons@University of Nebraska - Lincoln

2016

\title{
Frontal plane comparison between drop jump and vertical jump: Implications for the assessment of ACL risk of injury
}

\author{
Guilherme Manna Cesar \\ University of Nebraska-Lincoln, g.cesar@unf.edu \\ Curtis L. Tomasevicz \\ University of Nebraska - Lincoln, ctomasevicz2@unl.edu \\ Judith M. Burnfield \\ Madonna Rehabilitation Hospital, Lincoln, NE, jburnfield@madonna.org
}

Follow this and additional works at: https://digitalcommons.unl.edu/athleticresearch

Part of the Bioinformatics Commons, Biomechanics Commons, Biophysics Commons, Exercise

Science Commons, Motor Control Commons, and the Psychology of Movement Commons

Cesar, Guilherme Manna; Tomasevicz, Curtis L.; and Burnfield, Judith M., "Frontal plane comparison between drop jump and vertical jump: Implications for the assessment of ACL risk of injury" (2016). Athletic Performance Research. 5.

https://digitalcommons.unl.edu/athleticresearch/5

This Article is brought to you for free and open access by the Athletics at DigitalCommons@University of Nebraska - Lincoln. It has been accepted for inclusion in Athletic Performance Research by an authorized administrator of DigitalCommons@University of Nebraska - Lincoln. 


\title{
Frontal plane comparison between drop jump and vertical jump: Implications for the assessment of ACL risk of injury
}

\author{
Guilherme M. Cesar, ${ }^{1}$ Curtis L. Tomasevicz, ${ }^{2}$ and Judith M. Burnfield ${ }^{3}$ \\ 1 Movement and Neurosciences Center, Institute for Rehabilitation Science and Engineering, \\ Madonna Rehabilitation Hospital, Lincoln, NE \\ 2 Department of Biological Systems Engineering, College of Engineering, University of Nebraska- \\ Lincoln, Lincoln, NE \\ 3 Institute for Rehabilitation Science and Engineering, Madonna Rehabilitation Hospital, Lincoln, NE \\ Corresponding author — Guilherme M. Cesar, gcesar@madonna.org
}

\begin{abstract}
The potential to use the vertical jump (VJ) to assess both athletic performance and risk of anterior cruciate ligament $(\mathrm{ACL})$ injury could have widespread clinical implications since $\mathrm{VJ}$ is broadly used in high school, university, and professional sport settings. Although drop jump (DJ) and VJ observationally exhibit similar lower extremity mechanics, the extent to which VJ can also be used as screening tool for $\mathrm{ACL}$ injury risk has not been assessed. This study evaluated whether individuals exhibit similar knee joint frontal plane kinematic and kinetic patterns when performing VJs compared with DJs. Twentyeight female collegiate athletes performed DJs and VJs. Paired $t$-tests indicated that peak knee valgus angles did not differ significantly between tasks ( $p=0.419)$; however, peak knee internal adductor moments were significantly larger during the DJ vs. VJ $(p<0.001)$. Pearson correlations between the DJ and VJ revealed strong correlations for knee valgus angles $(r=0.93, p<0.001)$ and for internal knee adductor moments $(r=0.82, p<0.001)$. Our results provide grounds for investigating whether frontal plane knee mechanics during VJ can predict $A C L$ injuries and thus can be used as an effective tool for the assessment of risk of $A C L$ injury in female athletes.
\end{abstract}

Keywords: Risk of AC L injury, kinematics, kinetics, female athletes, lower extremity assessment

\section{Introduction}

Surgical repair and physical rehabilitation of anterior cruciate ligament (ACL) injuries are expensive and time-consuming, and often athletes have difficulty attaining pre-injury playing capacity (Ardern, Taylor, Feller, \& Webster, 2014; de Loes, Dahlstedt, \& Thomee, 2000; Hewett, Myer, \& Ford, 2006). Over the short term, the resulting deficits can detrimentally impact not only scholarship eligibility but also academic performance (Freedman, Glasgow, Glasgow, \& Bernstein, 1998). Orthopedic complications, such as meniscal tears (Hagino et al., 2015) and early onset knee osteoarthritis (Butler, Minick, Ferber, \& Underwood, 2009; Palmieri-Smith \& Thomas, 2009), are not uncommon. The numerous negative sequels of an ACL injury highlight the need to identify effective screening tools for ACL injury prevention. 
Non-contact ACL injuries have received considerable attention, in part, due to the clinical ability to screen for individuals who may be at greater risk and intervene prior to injury. Female athletes, in particular, exhibit a four to six-fold higher incidence of non-contact ACL injuries compared with their male counterparts performing the same landing or cutting sport (Arendt \& Dick, 1995). Across the multiple domains that have been studied (e.g. anatomical, hormonal, neuromuscular, and biomechanical), frontal plane knee joint collapse (i.e. dynamic knee valgus) and the consequential increased internal knee adductor moments during landing tasks have been identified as key biomechanical factors predisposing injury. For example, a cohort of female athletes who subsequently experienced ACL ruptures exhibited $8^{\circ}$ greater valgus during pre-screening landing maneuvers than non-injured athletes in a prospective study of 205 adolescent females across a season (Hewett et al., 2005). Visual analysis of 22 female basketball athletes' ACL injury videos (Krosshaug et al., 2007) corroborated that knee valgus collapse is frequently present during female athletes' injuries. Taken together, these findings illustrate the importance of screening for injurious knee movement patterns (e.g. valgus) to intensify preventive interventions.

In order to assess dynamic knee valgus and lower extremity mechanics for potential risks of ACL injury during landing, many double-limb and single-limb tasks have been used including the drop jump (DJ) (e.g. Stearns \& Powers, 2014), drop landing (e.g. Pollard, Sigward, \& Powers, 2010), and single-leg landing (e.g. Cesar et al., 2011). Landing tasks require the use of an apparatus (box or raised platform) for the assessment to be accomplished and this may not be feasible on a day-to-day basis considering the training environment and the volume of athletes to be assessed. Additionally, comparing results from the literature regarding lower extremity mechanics from fixed landing heights across athletes with different statures may provide misleading information as an athlete may experience different joint reaction forces with varying box heights (Bobbert, Huijing, \& van Ingen Schenau, 1987).

While the landing phase of the DJ places high moment demands around the knee joint and is regularly used for the assessment of dynamic knee valgus, the vertical jump (VJ) is the jumping maneuver commonly used in current protocols evaluating athletic performance (e.g. Nesser \& Lee, 2009). The VJ assessment in competitive athletic environment, as observed in the scouting combine for the National Football League (Keller et al., 2015; Robbins, Goodale, Kuzmits, \& Adams, 2013), is designed to challenge the athletes' capacity to generate lower extremity power and achieve maximal vertical jumping height. High moment demands around lower extremity joints are expected as athletes exert their best effort to successfully accomplish this task. Although similar lower extremity mechanics are anecdotally seen when individuals perform both DJ and VJ tasks, to date, the extent to which VJ can be used as a screening tool for dynamic knee valgus has not been assessed. In particular, it is conceivable that the pre-flight phase of the VJ task could also be used to assess the risk for ACL injuries if knee joint valgus angles and internal adductor moments were similar and correlated with the values observed during the landing phase of DJ. The potential to use VJ not only for athletic performance assessment, but also as a screening tool for risks of ACL injury, has a widespread clinical implication since this jumping task is broadly used in high school, university, and professional sport settings.

The purpose of the present study was to determine whether the pre-flight phase of VJ provides similar knee joint frontal plane kinematic and kinetic patterns when compared with the landing phase of the DJ. We compared the magnitude of knee joint valgus angles 
and internal adductor moments between both tasks. We hypothesized that knee joint valgus angles would not differ between tasks and that the values obtained from each test would positively correlate. In addition, we hypothesized that knee joint internal adductor moments would differ between tasks, given the presence of higher ground reaction forces during the DJ compared with VJ (Kollias, Panoutsakopoulos, \& Papaiakovou, 2004); however, the values obtained would positively correlate.

\section{Methods}

\section{Participants}

Thirty National Collegiate Athletic Association (NCAA) Division I female athletes participated in this study. Data from 28 participants were used for statistical treatment, and their mean $( \pm S D)$ age, height, and mass were $19.7 \pm 1.1$ years, $171.3 \pm 10.5 \mathrm{~cm}$, and $68.7 \pm 8.3 \mathrm{~kg}$, respectively. Participants were involved in soccer $(n=15)$, volleyball $(n=8)$, and gymnastics $(n=5)$. Participants were healthy at the time of data collection with no history of lower extremity or trunk injuries in the prior six months. Exclusion criteria involved previous injury that resulted in ligamentous laxity at the hip, knee, or ankle joints, and presence of any medical or neurologic condition (e.g. concussion) that would impair the current ability to perform athletic maneuvers. Study procedures were explained to each student-athlete and informed consent and parental assent (when required) were obtained before participation in accordance with the University of Nebraska Institutional Review Board.

\section{Instrumentation}

An 11-camera motion analysis system (Qualisys®, Gothenburg, Sweden) captured threedimensional kinematic data at $250 \mathrm{~Hz}$. Kinetic data were obtained using two $0.90 \mathrm{~m} \times 0.90$ $\mathrm{m}$ force platforms (Bertec Corporation, Columbus, OH, USA) sampled at 1,500 Hz.

\section{Procedures}

Testing took place at the Nebraska Athletic Performance Laboratory. Participants wore selfselected athletic footwear. Twenty-three reflective markers were placed over anatomical landmarks to reconstruct the three-dimensional movement of the participants' pelvis and lower extremity segments. Markers were placed bilaterally over the distal end of second and fifth metatarsal bones (on the shoe), heel (on the shoe), medial and lateral malleoli, tibial tuberosity, medial and lateral epicondyles of femur, greater trochanters, iliac crests, L5S1 junction, and a marker placed above the patella (one-third of the distance between patella and anterior superior iliac spine).

The experimental tasks used in this study were performed as part of a larger data collection protocol that included three other performance tasks. The two jumping tasks analyzed in this study were the first two tests of the protocol after the seven-min warm-up session, which included jogging drills and dynamic stretching to full range of motion under the supervision of the team's respective strength and conditioning coach. For each task, participants performed practice trials for familiarization of procedures and instrumentation. Task order was randomly assigned with 14 participants performing VJ first followed 
by the DJ, and 14 participants performing the DJ as the first task. All participants were familiar with both DJ and VJ activities, given their previous experiences in the athletic environment. Three trials were recorded for each task and they were not provided with any verbal cues for landing or jumping technique.

For the VJ task, participants stood with both arms raised overhead and their dominant hand closest to the Vertec device (Power Systems, Knoxville, TN, USA). Their feet were placed shoulder-width apart, with each foot on a separate force plate. Participants held this position for one second, and were then instructed to perform a single counter-movement and jump straight vertically to reach maximal height by targeting the highest possible vane on the Vertec with their dominant hand. The depth of the counter-movement was not controlled and each participant performed the counter-movement to their comfort.

For the DJ task, participants were instructed to step off the 40-cm platform with their preferred leg, land on the floor with both legs, and immediately jump straight vertically to reach maximal height by targeting the highest possible vane on the Vertec. Motion of the upper extremity was not controlled and each participant was free to use their arms as a counter-movement to increase their jumping performance. Since they were not instructed to aim at the force plates for the landing, the athletes who did not land with each foot on a separate force plate were not included in this study $(n=2)$.

\section{Data processing}

Kinematic and kinetic data were processed in Visual3D ${ }^{\mathrm{TM}}$ (C-Motion, Inc., Rockville, MD, USA) and custom Matlab (MathWorks, Natick, MA, USA) codes were used to identify variables of interest. Marker trajectory and force data were filtered using a fourth-order Butterworth low-pass filter with a 10-Hz cut-off frequency.

Local coordinate systems for the body segments (i.e. pelvis, thighs, shanks, and feet) were derived from the standing calibration trial. Six degrees of freedom of each segment were determined from the segment's kinematic triad by transforming the triad of markers to the position and orientation of each segment determined from the standing calibration trial. Joint kinematics were calculated using a joint coordinate approach (Grood \& Suntay, 1983).

Internal net joint moments were calculated using standard inverse dynamics equations (Grood \& Suntay, 1983) and were normalized to body mass and height (Moisio, Sumner, Shott, \& Hurwitz, 2003). Segmental masses were assigned based on the anthropometric data of Dempster (Dempster, 1955).

Positive value $(+)$ was used to report knee valgus angle and internal adductor moment. For VJ, variables of interest were calculated in the pre-flight phase, determined as the time between the initial downward motion of the L5-S1 marker and the time when both feet left the force plates (toe-off). For DJ, variables of interest were calculated during the landing phase, determined as the time between initial contact with the force plate (stipulated as an increase in $10 \mathrm{~N}$ of the vertical force) and toe-off. These events were determined using a position-based and force-based detection algorithm and verified by visual inspection.

\section{Statistical analysis}

The dependent variables considered for this study included peak knee valgus angle (kinematics) and peak internal adductor moment (kinetics). Descriptive statistics were calculated 
and expressed as $M, S D$, minimum, and maximum values for each variable of interest. Paired sample $t$-tests were used to detect differences in all dependent variables. Pearson correlation coefficients $(r)$ were used to examine the relationship of the dependent variables between both tasks. Observed power (OP; $1-\beta)$ was calculated to evaluate the strength of the drawn inferences regarding both statistical treatments. Variables were identified during each of the three trials for both DJ and VJ tasks and the trial with the highest recorded value (i.e. angle or moment) was selected for statistical analysis for each participant. All statistical analyses were performed (SPSS Inc., Chicago IL, USA) with significant levels set a priori at $\alpha=0.05$.

\section{Results}

Descriptive statistics of peak knee joint valgus angle and peak internal adductor moment obtained from each task are provided in Table 1.

Peak knee valgus angle did not differ significantly between DJ and VJ ( $p=0.419$; OP = 0.93). The peak knee valgus angle during DJ correlated very strongly with VJ's peak knee valgus angle $(r=0.93 ; p<0.001 ; \mathrm{OP}>0.99$; Figure 1$)$.

Peak knee joint internal adductor moment value obtained from DJ was significantly larger than VJ $(p<0.001 ; \mathrm{OP}>0.99)$. The internal adductor moment from DJ exhibited a strong correlation with VJ internal adductor moment $(r=0.82 ; p<0.001 ; \mathrm{OP}>0.99$; Figure 2$)$.

Table 1. Descriptive statistics of peak knee joint valgus angle and internal adductor moment for the DJ and VJ tasks.

\begin{tabular}{|c|c|c|c|c|c|c|}
\hline & \multicolumn{2}{|c|}{$M \pm S D$} & \multicolumn{2}{|c|}{ Minimum } & \multicolumn{2}{|c|}{ Maximum } \\
\hline & DJ & VJ & DJ & VJ & DJ & VJ \\
\hline Knee valqus angle $\left(^{\circ}\right)$ & $9.7 \pm 3.2$ & $9.5 \pm 2.9$ & 2.8 & 4.6 & 16.8 & 16.7 \\
\hline Knee internal adductor moment $(\mathrm{N} / \mathrm{kgm})$ & $0.26 \pm 0.10$ & $0.21 \pm 0.08$ & 0.05 & 0.04 & 0.45 & 0.33 \\
\hline
\end{tabular}

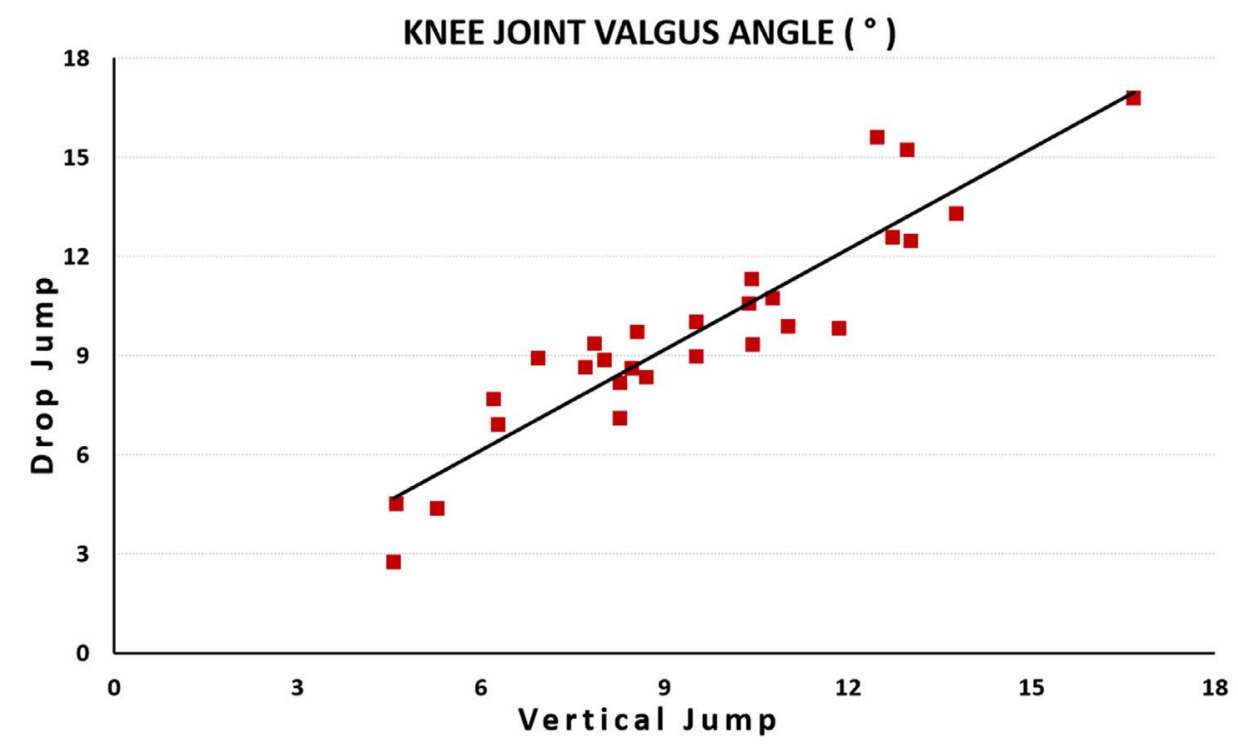

Figure 1. Significant peak knee valgus angle relationship $(p<0.001 ; r=0.93)$ between DJ and VJ. 




Figure 2. Significant peak knee joint internal adductor moment relationship $(p<0.001 ; r=0.82)$ between DJ and VJ.

\section{Discussion and implications}

Screening to identify athletes at greater risk for non-contact ACL injuries is key to enhance prevention efforts and to athletes' overall long-term health and well-being. Currently, athletes of all ages undergo frequent screenings for athleticism using the VJ, while DJ screens are conducted less frequently. The current research explored whether the VJ could be used not only as a tool to screen for changes in athleticism (e.g. Noyes, Barber-Westin, Smith, \& Campbell, 2013; Robbins et al., 2013), but also used as a potential tool to identify individuals at risk for non-contact ACL injuries since our sample exhibited similar lower extremity mechanics when performing both tasks. Our results strongly suggest that VJ can be used for both purposes concurrently. The ubiquitous nature of the VJ could make it an appealing screening tool for coaching and medical staff.

Consistent with our initial hypothesis, the magnitude of knee valgus angle achieved during the VJ and DJ was similar for the female athletes assessed. The strong correlation observed between the knee valgus angle during the DJ (which is currently used as a clinical screen for ACL injuries) and the VJ $(r=0.93)$ further supports the feasibility of using VJ as a potential screening tool for the risk of non-contact ACL injuries in female athletes. It is important to note, however, that additional research is required to understand the extent to which the DJ assessment can be used to identify athletes at increased risk of suffering ACL injuries (Goetschius et al., 2012; Krosshaug et al., 2016; Smith et al., 2012). The findings of our study provide support for investigating whether frontal plane knee mechanics during VJ can predict ACL injury and thus be used as an injury screening tool.

Clearly, advantages of the VJ task are that it is routinely performed across different environments, such as high school (e.g. Noyes et al., 2013), collegiate (e.g. Nesser \& Lee, 2009; Robbins et al., 2013), and professional settings (e.g. Keller et al., 2015). It is often performed several times within an athlete's season, thus allowing for the evaluation of individual 
responses to training programs and other injuries across the sports continuum and the athlete's career. This would facilitate time-efficient and consistent monitoring of athletes' safety regarding potential risks for ACL injury.

Although the current study used expensive three-dimensional motion analysis technology to perform the DJ and VJ evaluations, this may not be requisite for clinical analyses. Visual inspection of knee valgus with an associated score either live or from a camera capturing images of the frontal plane could serve as an alternative approach (Nilstad et al., 2014). Although not as accurate as three-dimensional motion analysis, visual screening provides a reliable and valid (intra-rater average $\kappa$ value 0.80 [0.65-1.0]; interrater average $\kappa$ value 0.79 [0.61-0.97]) option when three-dimensional systems are not available to evaluate knee valgus (Ekegren, Miller, Celebrini, Eng, \& Macintyre, 2009).

While studies usually investigate knee valgus collapse within the absorption phase of the DJ (i.e. from initial contact to peak knee flexion), we presented the peak knee valgus angle (and its correspondent moment data) that occurred within the entire task (i.e. from initial contact to toe-off). This time frame was chosen based on current literature (Nilstad et al., 2014) to facilitate the application of our study to sport clinicians and coaching staff. In order to detect whether valgus angle occurred prior to peak knee flexion, one would need to rely on three-dimensional motion analysis systems or multiple synchronized video cameras placed around the athlete. Given that peak valgus angles are similar between VJs and DJs in female athletes, screening for potential risks of non-contact ACL injury could be simplified for this population by using visual observation techniques (Ekegren et al., 2009) or two-dimensional video analysis (e.g. Dartfish, Fribourg, Switzerland) and products alike to screen for potential risks of non-contact ACL injury.

Consistent with our final hypothesis, larger adductor moments were observed during the DJ compared with VJ (despite the similarity of valgus angles across tasks). The strong positive correlation $(r=0.82)$ observed for the moment data indicates that individuals likely to collapse into excess valgus during the DJ would also be expected to do so during the VJ. The apparent disparity in adductor moment magnitude between DJ and VJ $(0.26 \mathrm{~N} / \mathrm{kg} \cdot \mathrm{m}$ vs $0.21 \mathrm{~N} / \mathrm{kg} \cdot \mathrm{m}$, respectively), however, is not surprising, given unique characteristics of each task. For example, during the VJ, participants start with their feet on the ground and the knees rotate into valgus as each lower limb rapidly generates the forces required to explosively lift the body from the ground towards the targeted vanes. During the DJ, however, gravity accelerated the body mass downward through the $40-\mathrm{cm}$ fall from the platform leading to larger impact forces as the athletes decelerated their bodies during the landing phase. These larger forces were confirmed in a post hoc analysis as the peak vertical ground reaction force normalized to body mass was significantly greater $(p<0.001)$ during DJ (3.39 $\pm 0.52 \mathrm{~N} / \mathrm{kg})$ than VJ $(2.68 \pm 0.44 \mathrm{~N} / \mathrm{kg})$. Given the position of the knee joint (i.e. valgus) during DJ, larger adductor moments were experienced by the athletes in the frontal plane. It is important to consider that the greater peak ground reaction force could be due to either the impact with the ground or greater push-off from the ground to jump during the DJ task.

Our results have significant clinical implications during athletic performance screening procedures within collegiate settings. Taking into account limits placed by the NCAA on contact time between coaching staff and student-athletes, the use of one test to detect both athleticism and potential risks of ACL injuries is key to efficiently address important measurements for strength and conditioning coaches while providing robust data for the athletic medicine staff. 


\section{Limitations and continuing work}

Our data provide a foundation for future studies considering efficient manners to measure athleticism and the impact of training in high school, collegiate, and professional athletes while simultaneously addressing a key feature of the athlete's safety: lower extremity movement patterns related to ACL risk of injury. Although literature questions the reliability of quantifying knee joint rotations with skin-mounted markers (Benoit et al., 2006; Leardini, Chiari, Della Croce, \& Cappozzo, 2005; Reinschmidt, van den Bogert, Nigg, Lundberg, \& Murphy, 1997), we showed that clinically relevant kinematic and kinetic variables recorded with the DJ and VJ display similar patterns across a cohort of female collegiate athletes. However, it is not yet known whether values recorded with the VJ are predictive of injuries. Thus, additional work is required to understand if this relationship also exists in male collegiate athletes and to determine the extent to which VJ can be used as a predictor for ACL injuries across both sexes. Additionally, future work exploring similarities between sagittal plane kinematics (e.g. hip and knee flexion angles) and kinetics (i.e. extensor moments) could provide valuable insights into the two tasks' demands and the relationship to potential risk for ACL injury.

\section{Conclusion}

When compared with the DJ, the VJ exhibits a similar knee joint frontal plane kinematic pattern and its frontal plane kinematics and kinetics are strongly correlated with the pattern observed during DJ in female athletes. The DJ task is extensively used for the assessment of risk of ACL injury. Since VJ has a widespread use for the evaluation of athleticism, utilizing this task concurrently for the potential evaluation for the risk of ACL injury would broaden testing for athletes' safety and well-being.

\section{References}

Ardern, C. L., Taylor, N. F., Feller, J. A., \& Webster, K. E. (2014). Fifty-five per cent return to competitive sport following anterior cruciate ligament reconstruction surgery: An updated systematic review and meta-analysis including aspects of physical functioning and contextual factors. British Journal of Sports Medicine, 48, 1543-1552.

Arendt, E., \& Dick, R. (1995). Knee injury patterns among men and women in collegiate basketball and soccer: NCAA data and review of literature. The American Journal of Sports Medicine, 23, 694-701.

Benoit, D. L., Ramsey, D. K., Lamontagne, M., Xu, L., Wretenberg, P., \& Renström, P. (2006). Effect of skin movement artifact on knee kinematics during gait and cutting motions measured in vivo. Gait and Posture, 24, 152-164.

Bobbert, M. F., Huijing, P. A., \& van Ingen Schenau, G. J. (1987). Drop jumping. II. The influence of dropping height on the biomechanics of drop jumping. Medicine and Science in Sports and Exercise, 19, 339-346.

Butler, R. J., Minick, K. I., Ferber, R., \& Underwood, F. (2009). Gait mechanics after ACL reconstruction: Implications for the early onset of knee osteoarthritis. British Journal of Sports Medicine, 43, 366-370.

Cesar, G. M., Pereira, V. S., Santiago, P. R., Benze, B. G., da Costa, P. H., Amorim, C. F., \& Serrão, F. V. (2011). Variations in dynamic knee valgus and gluteus medius onset timing in non-athletic females related to hormonal changes during the menstrual cycle. The Knee, 18, 224-230. 
de Loes, M., Dahlstedt, L. J., \& Thomee, R. (2000). A 7-year study on risks and costs of knee injuries in male and female youth participants in 12 sports. Scandinavian Journal of Medicine and Science in Sports, 10, 90-97.

Dempster, W. T. (1955). Space requirements of the seated operator. Geometrical, kinematic, and mechanical aspects of the body with special reference to the limbs. In WADC Technical Report 55-159. Wright-Patterson Air Force Base, Carpenter Litho \& Printing Co., Springfield, OH.

Ekegren, C. L., Miller, W. C., Celebrini, R. G., Eng, J. J., \& Macintyre, D. L. (2009). Reliability and validity of observational risk screening in evaluating dynamic knee valgus. Journal of Orthopaedic $\mathcal{E}$ Sports Physical Therapy, 39, 665-676.

Freedman, K. B., Glasgow, M. T., Glasgow, S. G., \& Bernstein, J. (1998). Anterior cruciate ligament injury and reconstruction among university students. Clinical Orthopaedics and Related Research, $356,208-212$.

Goetschius, J., Smith, H. C., Vacek, P. M., Holterman, L. A., Shultz, S. J., Tourville, T. W., ... Beynnon, B. D. (2012). Application of a clinic-based algorithm as a tool to identify female athletes at risk for anterior cruciate ligament injury: A prospective cohort study with a nested, matched case-control analysis. The American Journal of Sports Medicine, 40, 1978-1984.

Grood, E. S., \& Suntay, W. J. (1983). A joint coordinate system for the clinical description of three-dimensional motions: Application to the knee. Journal of Biomechanical Engineering, 105, 136-144.

Hagino, T., Ochiai, S., Senga, S., Yamashita, T., Wako, M., Ando, T., \& Haro, H. (2015). Meniscal tears associated with anterior cruciate ligament injury. Archives of Orthopaedic and Trauma Surgery, 135, 1701-1706.

Hewett, T. E., Myer, G. D., \& Ford, K. R. (2006). Anterior cruciate ligament injuries in female athletes: Part 1, mechanisms and risk factors. American Journal of Sports Medicine, 34, 299-311.

Hewett, T. E., Myer, G. D., Ford, K. R., Heidt Jr, R. S., Colosimo, A. J., McLean, S. G., ... Succop, P. (2005). Biomechanical measures of neuromuscular control and valgus loading of the knee predict anterior cruciate ligament injury risk in female athletes: A prospective study. American Journal of Sports Medicine, 33, 492-501.

Keller, R. A., Mehran, N., Austin, W., Marshall, N. E., Bastin, K., \& Moutzouros, V. (2015). Athletic performance at the NFL scouting combine after anterior cruciate ligament reconstruction. The American Journal of Sports Medicine, 43, 3022-3026.

Kollias, I., Panoutsakopoulos, V., \& Papaiakovou, G. (2004). Comparing jumping ability among athletes of various sports: Vertical drop jumping from $60 \mathrm{~cm}$. Journal of Strength and Conditioning Research, 18, 546-550.

Krosshaug, T., Nakamae, A., Boden, B. P., Engebretsen, L., Smith, G., Slauterbeck, J. R., ... Bahr, R. (2007). Mechanisms of anterior cruciate ligament injury in basketball: Video analysis of 39 cases. American Journal of Sports Medicine, 35, 359-367.

Krosshaug, T., Steffen, K., Kristianslund, E., Nilstad, A., Mok, K. M., Myklebust, G., ... Bahr, R. (2016). The vertical drop jump is a poor screening test for ACL injuries in female elite soccer and handball players: A prospective cohort study of 710 Athletes. American Journal of Sports Medicine, 44, 874-883.

Leardini, A., Chiari, L., Della Croce, U., \& Cappozzo, A. (2005). Human movement analysis using stereophotogrammetry. Part 3. Soft tissue artifact assessment and compensation. Gait and Posture, 21, 212-225.

Moisio, K. C., Sumner, D. R., Shott, S., \& Hurwitz, D. E. (2003). Normalization of joint moments during gait: A comparison of two techniques. Journal of Biomechanics, 36, 599-603.

Nesser, T. W., \& Lee, W. L. (2009). The relationship between core strength and performance in division I female soccer players. Journal of Exercise Physiology Online, 12, 21-28.

Nilstad, A., Andersen, T. E., Kristianslund, E., Bahr, R., Myklebust, G., Steffen, K., \& Krosshaug, T. (2014). Physiotherapists can identify female football players with high knee valgus angles during 
vertical drop jumps using real-time observational screening. Journal of Orthopaedic \& Sports Physical Therapy, 44, 358-365.

Noyes, F. R., Barber-Westin, S. D., Smith, S. T. T., \& Campbell, T. (2013). A training program to improve neuromuscular and performance indices in female high school soccer players. Journal of Strength \& Conditioning Research, 27, 340-351.

Palmieri-Smith, R. M., \& Thomas, A. C. (2009). A neuromuscular mechanism of posttraumatic osteoarthritis associated with ACL injury. Exercise and Sport Sciences Reviews, 37, 147-153.

Pollard, C. D., Sigward, S. M., \& Powers, C. M. (2010). Limited hip and knee flexion during landing is associated with increased frontal plane knee motion and moments. Clinical Biomechanics, 25, 142-146.

Reinschmidt, C., van den Bogert, A. J., Nigg, B. M., Lundberg, A., \& Murphy, N. (1997). Effect of skin movement on the analysis of skeletal knee joint motion during running. Journal of Biomechanics, 30, 729-732.

Robbins, D. W., Goodale, T. L., Kuzmits, F. E., \& Adams, A. J. (2013). Changes in the athletic profile of elite college American football players. Journal of Strength E Conditioning Research, 27, 861-874.

Smith, H. C., Johnson, R. J., Shultz, S. J., Tourville, T., Holterman, L. A., Slauterbeck, J., ... Beynnon, B. D. (2012). A prospective evaluation of the landing error scoring system (LESS) as a screening tool for anterior cruciate ligament injury risk. The American Journal of Sports Medicine, 40, 521-526.

Stearns, K. M., \& Powers, C. M. (2014). Improvements in hip muscle performance result in increased use of the hip extensors and abductors during a landing task. The American Journal of Sports Medicine, 42, 602-609. 